\title{
High Moisture Sensitivity of the Elements Based on Carbon Nanotubes Array
}

\author{
Sergei Bulyarsky ${ }^{1}$, Vyacheslav Galperin ${ }^{2}$, Levan Ichkitidze ${ }^{3 *}$, Michael Ermakov ${ }^{1}$, Alexander Pavlov ${ }^{4}$, \\ Yuri Shaman ${ }^{2}$
}

${ }^{1}$ Ulyanovsk State University, Ulyanovsk, Russia; ${ }^{2}$ Scientific Manufacturing Complex "Technological Centre", Moscow, Russia; ${ }^{3}$ National Research University of Electronic Technology (MIET), Moscow, Russia; ${ }^{4}$ Institute of Nanotechnology of Microelectronics RAS, Moscow, Russia.

Email: *leo852@Inbox.ru

Received January $29^{\text {th }}, 2013$; revised March $27^{\text {th }}, 2013$; accepted April $14^{\text {th }}, 2013$

Copyright (C) 2013 Sergei Bulyarsky et al. This is an open access article distributed under the Creative Commons Attribution License, which permits unrestricted use, distribution, and reproduction in any medium, provided the original work is properly cited.

\begin{abstract}
The results of the research and development of the moisture-sensitive elements based on the carbon nanotubes (CNT) array are presented. It was shown that CNT arrays that were grown by low-temperature plasma enhanced chemical vapor deposition (PECVD) method on the planar Si structures exhibit extremely high moisture sensitivity. The structure resistance ratio in dry and moisture conditions exceed 400 . Such relatively high change in resistances is conditioned by the pattern of change of the charge carrier's conductivity between certain nanotubes in the bundle when water molecules adsorption occurs.
\end{abstract}

Keywords: Carbon Nanotubes; Moisture-Sensitive Elements; Conductivity; Molecules Adsorption

CNT possess a number of unique properties including high sorption capacity of the various nature molecules [1]. Water absorption of the carbon structures has a number of features because of high interactions between the water molecules and relatively weak interaction between the water and carbon [2]. The calculation shows that in the pores formed by adjacent nanotubes in the bundle there are the chains of water molecules near the nanotubes surface [2] which in turn should lead to the changes of the CNT electronic structure [3]. Such features are fully manifested in CNT arrays, creating a high sensitivity to moisture.

This article presents the results of the fabrication and study the moisture-sensitive elements based on CNT arrays. The vertical arrangement of the nanotubes in the array that was grown on the planar Si structure combined with a current flow with perpendicular direction to the CNT axis allowed us to obtain high resistance ratio of the structure in the dry and moisture conditions which is more then 400 times.

CNT arrays synthesis was performed by PECVD method at $500^{\circ} \mathrm{C}$ for $20 \mathrm{~min}$. $\mathrm{FeNiCo}_{20}$ film $5 \mathrm{~nm}$ thick deposited on the Ti buffer layer $50 \mathrm{~nm}$ thick was used as the growth catalyst. Such film was prepared on the planar Si structure surface.

"Corresponding author.
Current conducting electrodes were made from polysilicon. Before the CNT array synthesis the catalyst was subjected to oxygen annealing at a substrate temperature $280^{\circ} \mathrm{C}$ for 5 minutes. CNT array that was obtained in this process is shown in Figure 1.

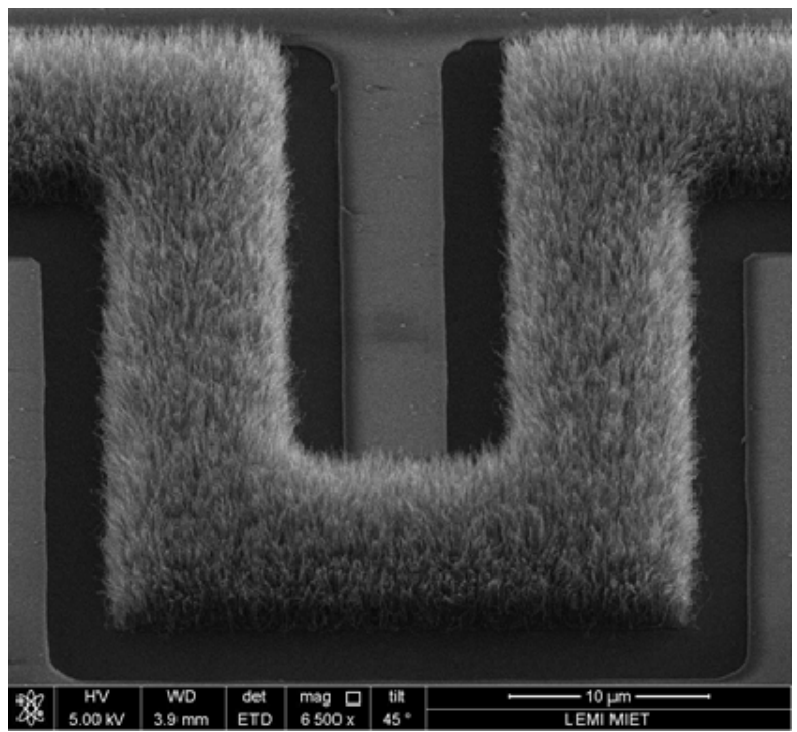

Figure 1. CNT array of the moisture-sensitive element. 
The average nanotubes high in the array was $2.5 \mu \mathrm{m}$. Catalyst sublayer looked like meander. The contact to CNT array was done by a catalyst sublayer and by side contacts from polysilicon tightly adjacent to array by means of features of the substrate relief. Before the measurements unoxidized catalyst residues were burned out by short current impulse. After the last operation current magnitude dramatically dropped, and the voltagecurrent characteristics became nonlinear instead of ohmic law.

Before the measurements, samples were dried in dry oxygen at $200^{\circ} \mathrm{C}$ for at least 3 hours. Then, in the dark at room temperature the voltage-current characteristic was measured. First measurement after above-mentioned drying mode showed high resistance samples with non-linear voltage-current characteristic. Before the second measurements the samples were placed into tinted closed vessel directly above the water surface. With identical voltage at room temperature current increase $10^{5}$ times. After the several cycling (drying-measurements in dry atmosphere-measurements in moisture atmosphere) it was obtained stable, repeatable voltage-current characteristic shown in Figure 2.

Continuous cyclic changes in the experiment conditions stabilized the current through the sample in dry and moist atmospheres. Such ratio decreased to 400 times still showing high sensitivity to moisture.

Such form of the voltage-current characteristic is well described by the formulas for tunnel recombination obtained in works $[4,5]$. In consistent with the results of these works, at specified voltage the transfer channel,

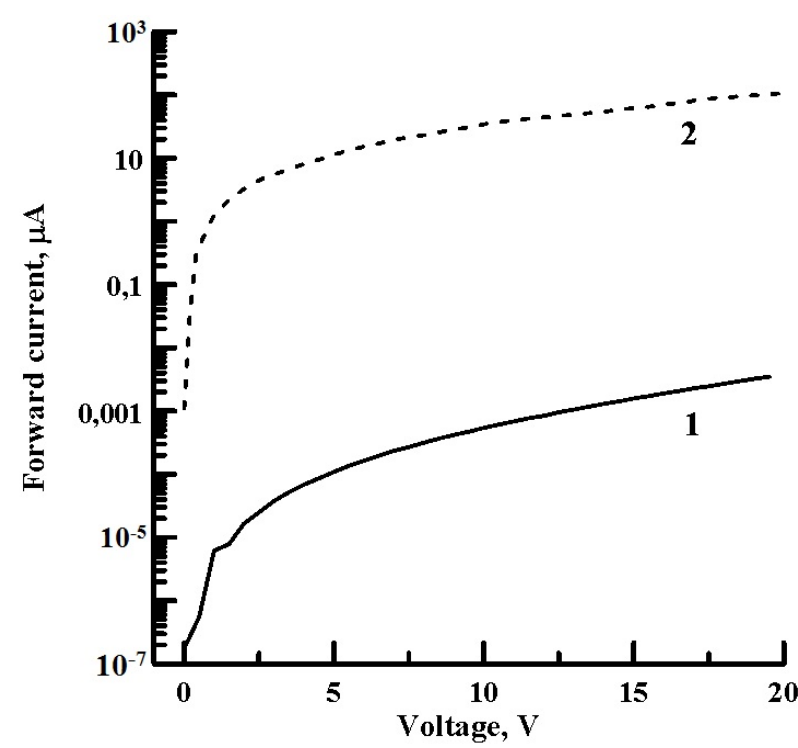

Figure 2. Voltage-current characteristic of the moisturesensitive element that was stabilized by the cyclic change of the experiment conditions. 1 , current value in dry atmosphere; 2 , current value in moist atmosphere. associated with tunnel or hopping transfer from a single localized state, saturates. Such limitation associated with a value of the density of states, between which there is an electron transition. Low density limits the current value.

In that cases when electron transfer from tube to tube is described by Mott hopping conductivity, probability of the tunneling transfer will be taken as the value that depends just on the overlap integral $[4,6]$ :

$$
w(E)=w_{L R}(E)=w_{R L}(E)=v \exp (-2 r / a),
$$

where $v$-frequency of attempts to overcame the potential barrier, equal to frequency of the characteristic phonon; $a=\hbar /(2 m E)^{1 / 2}$ — localization length [4], $r$ - overage jump distance equal to overage distance between the traps, which in turn defined by their concentration: $r=(N)^{-1 / 3}$.

In addition the current value is related to the concentration of the localized states $(N)$ by the formula $[4,5]$ :

$$
j_{r}=\frac{2 k_{B} T d(U)}{\left(U_{k}-U\right)} N^{2} v \exp \left[-\frac{2}{a(N)^{1 / 3}}\right],
$$

where $j_{r}$-is the current density at forward bias voltage $U ; U_{k}$ - contact potential difference; $S$ - (p-n-) junction area; $d(U)$ - width of the space-charge region, $k_{B}$-Boltzmann constant; $T$-temperature; $v$-the number of attempts per time unit of electron to overcome the potential barrier, which separates adjacent localized states.

The current ratio of the sensitive element in dry and moist atmosphere is associated with the concentration change of the localized states and with the localization length of the electron on the nanotube:

$$
\frac{j_{1}}{j_{2}}=\left(\frac{N_{1}}{N_{2}}\right)^{2} \exp \left(\frac{2}{a\left(N_{2}\right)^{1 / 3}}-\frac{2}{a\left(N_{1}\right)^{1 / 3}}\right),
$$

We can make the following assumptions about the causes of the significant increase in the conductivity of the tubes in a moist environment. Firstly, adsorption of water molecules is accompanied by a change in density of localized states [1] which leads to the increase in the current ratio in accordance with the Formula (3). Secondly, water molecules on the nanotube surface create the attractive interaction which in turn reduces the distance between nanotubes in the bundle. On this account the length of electron localization $a$ reduced, which is equal to overage distance that could be overcome by the electron when it transfers between adjacent nanotubes. This also leads to the increase in the current ratio.

Thus, in this study we have presented a new technology of growing CNT arrays, which essential point are two factors: the use of the solid catalyst film $\mathrm{FeNiCo}_{20}$, instead of gaseous ferrocene, which is usually used [1], and the oxidation of the catalyst before the synthesis of 
CNT array. It's experimentally shown that the movement of the electrons across the beam which is accompanied by their transition from one tube to another creates the conditions for significant change in the conductivity in the presence of water molecules adsorption. Such change can be explained by changes in the density of electronic states accompanying adsorption, and by the change in the distance between nanotubes in the bundle which impacts on the probability of electron transition between adjacent nanotubes.

\section{Acknowledgements}

We thank professors E. V. Blagov and S. V. Selishchev for their support of this research.

This work is partially funded by Ministry of Education of the Russian Federation (state contract No. 16.426. 11.0043 and 16.740.11.0765), and by Russian Fund Basic Research (state contract No. 10678p/19537).

\section{REFERENCES}

[1] S. Bulyarsky, "Carbon Nanotubes: Technology, Property
Control, Application," Ulyanovsk State University (Russia), Ulyanovsk, 2011, 478 pages. http://artlib.osu.ru/web/books/content_all/332.pdf

[2] A. Striolo, A. A. Chialvo, K. E. Gubbins and P. T. Cummings, "Water in Carbon Nanotubes: Adsorption Isotherms and Thermodynamic Properties from Molecular Simulation," Journal of Chemical Physics, Vol. 122, No. 23, 2005, Article ID: 234712. doi:10.1063/1.1924697

[3] O. B. Tomilin and U. U. Muryumin, "Carbon Nanotubes Adsorption on the Graphene Surface and Their Energy Spectrum," Solid State Physics (Russia), Vol. 48, No. 3, 2006, pp. 563-571.

[4] S. V. Bulyarsky, N. S. Grushko and A. Zhukov, "Calculating the Probability of Optical Transitions in Strong Electric Fields," Journal of Experimental and Theoretical Physics (Russia), Vol. 118, No. 11, 2000, pp. 1092-1098.

[5] S. V. Bulyarsky, Y. V. Rud, L. N. Vostrezova, A. S. Kagarmanov and O. A. Trifonov, "Tunneling Recombination in Semiconductor Structures with Nanodisorder," Physics and Technology of Semiconductors (Russia), Vol. 43, No. 4, 2009, pp. 460-466.

[6] N. Mott and A. Davis, "Electronic Processes in NonCrystalline Materials," Clarendon Press, Oxford, 1979, 590 pages. 